\title{
Thoracoscopic localization of intraparenchymal pulmonary nodules using direct intracavitary thoracoscopic ultrasonography prevents conversion of VATS procedures to thoracotomy in selected patients
}

\author{
Mohamed Khereba, MD, Pasquale Ferraro, MD, Andre Duranceau, MD, Jocelyne Martin, MD, MSc, \\ Eric Goudie, Vicky Thiffault, BScN, and Moishe Liberman, MD, PhD
}

\begin{abstract}
Objectives: To investigate the feasibility, accuracy, and effect on conversion rates of intracavitary video-assisted thoracoscopic surgery ultrasonography (VATS-US) for localization of difficult to visualize pulmonary nodules.

Methods: The study consisted of a prospective cohort of VATS-US for localization of intraparenchymal peripheral pulmonary nodules. Patients with pulmonary nodules not touching the visceral pleura on the computed tomography scan, who were scheduled for VATS wedge resection, were prospectively enrolled. The lobe of interest was examined: visually, using finger palpation when possible, and using the instrument sliding method. The nodule was then sought using a sterile ultrasound transducer. The primary outcome measure was the prevention of conversion to thoracotomy or lobectomy secondary to positive VATS-US findings in patients with nodules that were not identifiable using standard VATS techniques.
\end{abstract}

Results: Four different surgeons performed 45 individual VATS-US procedures during a 13-month period. Intracavitary VATS-US was able to detect 43 of 46 nodules. The sensitivity of VATS-US was $93 \%$, and the positive predictive value was $100 \%$. The lung nodules were visualized by thoracoscopic lung examination in 12 cases $(27 \%)$, palpable by finger in 18 cases $(40 \%)$, and palpable using the instrument sliding technique in 17 cases $(38 \%)$. In 20 cases, lung nodules were not identifiable using any of the traditional techniques and were identified only with VATS-US. VATS-US, therefore, prevented conversion to thoracotomy or lobectomy without tissue diagnosis in $43 \%(20 / 46)$ of cases.

Conclusions: Intracavitary VATS-US is a real-time, feasible, reliable, and effective method of localization of intraparenchymal pulmonary nodules during selected VATS wedge resection procedures and can decrease the conversion rates to thoracotomy or lobectomy. (J Thorac Cardiovasc Surg 2012;144:1160-6)

Thoracoscopic, nonanatomic wedge resection of nonsubpleural pulmonary nodules remains a challenge. This challenge is amplified when the nodules are small and/or deep in the pulmonary parenchyma. Video-assisted thoracoscopic surgery (VATS) has become the standard of care for resection of indeterminate pulmonary nodules for diagnosis, as the treatment of choice for pulmonary metastases, and for resection of primary lung cancer in selected high-risk patients with poor pulmonary function. ${ }^{1,2}$

From the Division of Thoracic Surgery, Department of Surgery, Centre Hospitalier de l'Université de Montréal, CHUM Endoscopic Tracheobronchial and Oesophageal Center, University of Montréal, Montréal, Québec, Canada.

Supported by the Canadian Foundation for Innovation and the Marcel and Rolande Gosselin Chair in Thoracic Surgical Oncology.

Disclosures: Authors have nothing to disclose with regard to commercial support.

Read at the 92nd Annual Meeting of The American Association for Thoracic Surgery, San Francisco, California, April 28-May 2, 2012.

Received for publication March 31, 2012; revisions received July 31, 2012; accepted for publication Aug 14, 2012; available ahead of print Sept 14, 2012.

Address for reprints: Moishe Liberman, MD, PhD, Division of Thoracic Surgery, Department of Surgery, Centre Hospitalier de l'Université de Montréal, CHUM Endoscopic Tracheobronchial and Oesophageal Center, University of Montréal, 1560 rue Sherbrooke Est, 8e CD - Pavillon Lachapelle, Bureau D-8051, Montréal, Québec H2L 4M1, Canada (E-mail: moishe.liberman@umontreal.ca).

0022-5223/\$36.00

Copyright (c) 2012 by The American Association for Thoracic Surgery

http://dx.doi.org/10.1016/j.jtcvs.2012.08.034
Traditional VATS techniques do not allow for bimanual lung palpation, making finding the nonsubpleural pulmonary nodule difficult. This is in sharp contrast to the open (thoracotomy) technique, which allows for bimanual palpation of the lung parenchyma and, therefore, typically, simple localization of nodules. Failure to localize small and relatively deep pulmonary nodules often leads to conversion to open thoracotomy. Conversion rates have been reported to be as great as $59 \%{ }^{3-5}$ Various preoperative and intraoperative techniques have been described for nodule localization during VATS. These techniques have included hook wire insertion, methylene blue injection, metallic coil insertion, technetium-99-labeled human serum albumin microspheres, and radiotracer-guided thoracoscopic biopsy. ${ }^{6-12}$

VATS-ultrasonography (VATS-US) is a simple, safe, and real-time method of pulmonary nodule localization during VATS procedures. We hypothesized that VATS-US could decrease the rate of conversion to thoracotomy in patients undergoing VATS wedge resection for small, nonsubpleural pulmonary nodules. The aims of the present study were to describe the sensitivity and specificity of VATS-US compared with traditional VATS techniques and the ability of VATS-US to prevent conversion to thoracotomy in patients with nodules not localized using traditional techniques. 


\section{Abbreviations and Acronyms \\ CT = computed tomography \\ VATS $=$ video-assisted thoracoscopic surgery \\ VATS-US $=$ video-assisted thoracoscopic surgery ultrasonography}

\section{MATERIALS AND METHODS}

The present study was a prospective clinical trial (clinicaltrials.gov identifier NCT01201824) evaluating VATS-US in a cohort of patients with nonsubpleural parenchymal pulmonary nodules. Each patient served as their own control. The primary outcome measure was the assessment of the validity, feasibility, and safety of surgeon-performed intraoperative intracavitary US as a localization technique for difficult to visualize pulmonary nodules during VATS procedures. The main secondary outcome was the ability of VATS-US to decrease the conversion rates of VATS procedures to open thoracotomy.

Other secondary outcomes included the following:

To compare the efficacy of VATS-US with other operative localization techniques for small pulmonary nodules during VATS (ie, visual localization, finger palpation, and the instrument sliding method).

To assess the ability of VATS-US to differentiate malignant versus benign pulmonary nodules using the US properties of the nodules.

To assess the ability of intraoperative intracavitary US to detect new pulmonary nodules, not detected on the preoperative computed tomography $(\mathrm{CT})$ scan.

The institutional review board at the Centre de Recherche de l'Université de Montreal approved the study. We screened all patients scheduled for VATS wedge resection for solitary or multiple pulmonary nodules. Eligible patients included those with CT scan-identified pulmonary nodules that were nonsubpleural (lung tissue between the nodule and visceral pleura on the preoperative CT scan) who were candidates for VATS resection. Patients with pulmonary nodules believed to be easily localized during VATS (large size, pleural retraction) were excluded from the present study. The eligible patients were prospectively enrolled after providing written informed consent.

The preoperative CT scan was used to record the size, site, and characteristics of the pulmonary nodules of interest and was used for comparison with the recorded values from the intraoperative VATS-US. The procedure date of the preoperative CT scan was recorded to calculate the interval between CT and VATS-US examination.

All procedures were performed with the patient under general anesthesia with single lung ventilation through double-lumen endotracheal intubation. Patient positioning, surgical preparation, and all surgical incisions were performed according to the standard VATS procedure of the surgeon performing the procedure. The surgeons were counseled not to change their typical VATS incisions (incision length, port size, position of incision) to accommodate the VATS-US probe or the angulation of the probe onto the lung.

After VATS port placement, the lung was completely deflated, and pleuroscopy was performed, searching for the nodule of concern using video thoracoscopy alone. The surgeon then tried to localize the nodule by finger palpation and then using the instrument sliding method.

A sterile, intracavitary, 10-mm VATS-US 5- to $10-\mathrm{MHz}$ linear probe with a flexible angulating tip (Aloka UST-5536-7.5; Hitachi Aloka Medical, Ltd, Hitachi, Japan; Figure 1) attached to an US processor (Aloka Alpha 10) was introduced through 1 of the VATS ports. The VATS-US examination was performed on the affected pulmonary lobe, attempting to localize the nodules of interest. Endoscopic articulation of the VATSUS probe often helps to identify nodules in more posterior or inferior locations. Sterile US jelly or water was used to help localize nodules not easily located with the initial US attempts.

The nodules were localized by direct US visualization of the nodule of concern (Figure 2) with or without the presence of a hyperechoic shadow underneath the nodule within the lung parenchyma (Figure 3). After nodule localization, the US properties, including size, distance from pleura, echogenicity, shape, sharpness of borders, and vascularity by Doppler examination, were recorded. After nodule localization, VATS wedge resection was performed. The resected lung tissue was macroscopically examined by the surgeon in the operating room to identify the resected lung nodules before sending the specimen for frozen section examination. In patients in whom the nodule of interest was not identified using any of the standard VATS techniques or by VATS-US, open thoracotomy was performed.

Four general thoracic surgeons performed or supervised all procedures in the present study. Three of these surgeons had no formal training in US or experience with US before the present study.

All final pathologic reports were reviewed to confirm the presence of the nodule of interest in the resected specimen and its nature. The pathologic reports were also reviewed for surgical margin status.

\section{RESULTS}

During a 13-month period (September 2010 to October 2011), 43 patients, including 21 men and 22 women, were prospectively enrolled in the present study. Patient age ranged from 28 to 88 years (mean, 57.7). VATS-US was used in 45 procedures. Two patients underwent surgery twice during the study, on bilateral sides, at an interval of 3 and 4 months. One patient underwent surgery for 2 nodules on the same side. Each of the 2 nodules was evaluated using VATS-US, and the patient underwent 2 VATS resections during the same procedure. The procedure indications included 34 performed for a preoperative diagnosis of a solitary pulmonary nodule, 10 for multiple pulmonary nodules, and 1 for a bronchogenic cyst.

The procedural time ranged from 24 to 163 minutes (mean, $73.4 \pm 34.0$ ). No major intraoperative complications occurred.

The mean interval between the preoperative $\mathrm{CT}$ scan and procedure was 84 days (median, 71). A total of 46 nodules were examined using VATS-US: 14 in the right lower lobe; 10 , right upper lobe; 2 , right middle lobe; 10 , left upper lobe; and 10, left lower lobe. The mean VATS-US-measured dimensions were $11.3 \mathrm{~mm}$ in the long axis (range, 2.7-27.7) and $8.6 \mathrm{~mm}$ in the short axis (range, 2-20.4). The distance from the visceral pleura was 1 to $24.1 \mathrm{~mm}$ (Table 1).

The site of all nodules found using VATS-US correlated with the preoperative CT scan findings. No additional nodules were identified by VATS-US that were not found on the preoperative $\mathrm{CT}$ scan.

Intracavitary VATS-US was able to detect $43(93.5 \%)$ of 46 nodules. VATS-US was unable to localize 3 pulmonary nodules (Table 2). The VATS-US procedural time ranged from less than 1 to 13 minutes (mean, $4.07 \pm 3.99$ ).

Of the 3 patients with nondetectable pulmonary nodules using VATS-US, 1 had Hodgkin's lymphoma with multiple indeterminate pulmonary nodules. The lung parenchyma was extremely edematous and thick, and the nodules were 


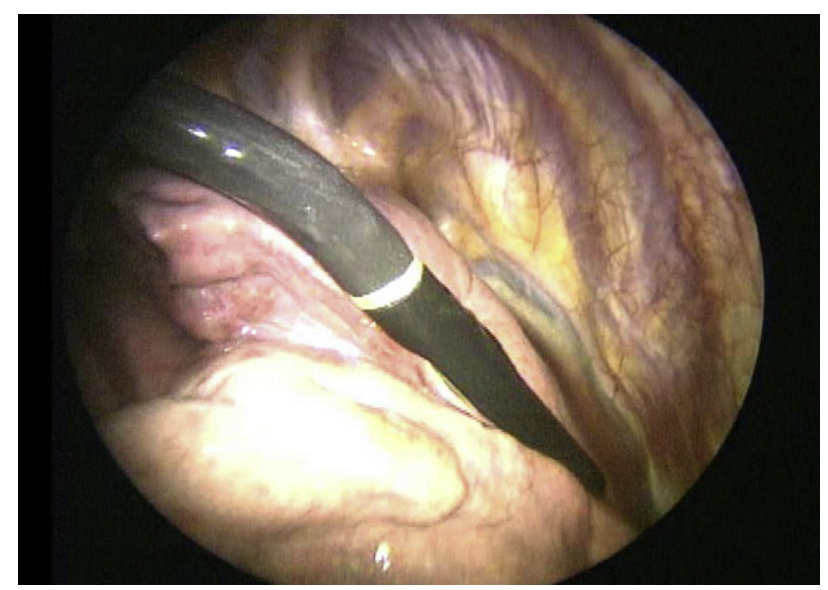

FIGURE 1. Intracavitary, 10-mm video-assisted thoracoscopic surgery ultrasonography, 5- to $10-\mathrm{MHz}$ linear probe with a flexible angulating tip (Aloka UST-5536-7.5).

not identifiable using US. The nodules were also not identifiable using VATS observation, finger palpation, or the instrument sliding technique. In this patient, blind wedge resection was performed using the preoperative CT scan images, and a nodule was found in the specimen. Pathologic examination confirmed the presence of the nodule, with a histologic diagnosis of pulmonary Hodgkin's lymphoma. One nodule that was not visible by VATS examination nor by VATS-US, was, however, palpable using finger palpation. The third nodule was extremely difficult to examine using VATS-US owing to a very posterior location very close to the spine. Because of the high probability of malignancy in the nodule (by CT and positron emission tomography scans), VATS lobectomy was performed. The nodule was easily detected using VATS-US ex vivo. In 1 patient, single lung ventilation was not possible owing to hypoxia. In this patient, VATS-US was able to localize the pulmonary nodule without lung isolation.
The sensitivity of VATS-US was $93.5 \%$, and the positive predictive value was $100 \%$. Lung nodules were visualized by thoracoscopic lung examination in 12 cases $(27 \%)$, palpable by finger in 18 nodules ( $40 \%$ ), and palpable using the instrument sliding technique in 17 nodules (38\%). Overlap was present because some nodules were localizable using more than 1 technique. The mean CT scan diameter for the 20 lesions not detected using standard intraoperative techniques was $14.5 \mathrm{~mm}$ (long axis) and $12.3 \mathrm{~mm}$ (short axis). The mean distance from the pleura was $11.0 \mathrm{~mm}$. Figure 4 compares the VATS localization technique ability to identify the nodule of interest in the study cohort.

The results were confirmed by pathologic examination, with negative margins in $100 \%$ of the pulmonary wedge resections. Pathologic analysis of the resected 46 nodules confirmed the presence of 31 malignant and 15 benign nodules. No significant differences were seen between the echogenic properties and final pathologic findings of the resected nodules (Table 3 ).

In 20 procedures ( 20 nodules), the lung nodules were not identifiable using any of the standard VATS techniques and were only found using VATS-US. VATS-US, therefore, prevented conversion to thoracotomy or lobectomy without tissue diagnosis in $43.5 \%$ (20/46) of cases. Furthermore, VATS-US confirmed nodule location before wedge resection in cases in which the location of the nodule was believed to be identified using traditional techniques.

\section{DISCUSSION}

VATS-US allows fast, real-time, and sensitive pulmonary nodule localization. It has the advantage of not requiring preoperative localization techniques and is, therefore, more convenient, efficient, safe, and less costly than techniques that require a separate procedure before VATS for nodule localization. It also avoids the logistical issues with the timing of multiple procedures and, in the present

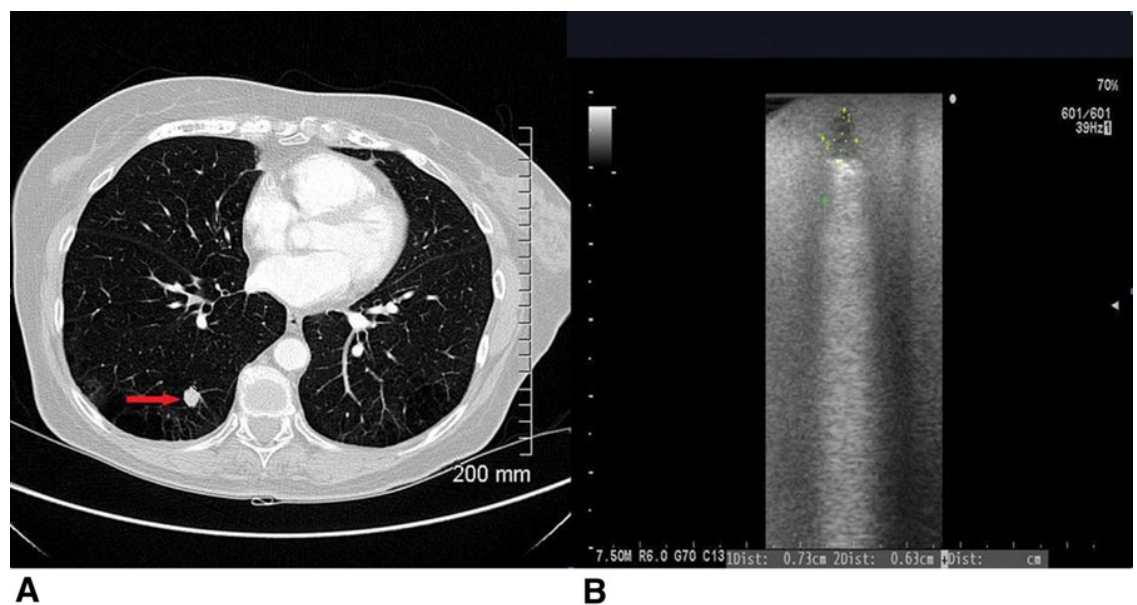

FIGURE 2. A, Small, 10-mm pulmonary nodule (arrow) located in the right lower lobe on preoperative computed tomography image. B, Corresponding video-assisted thoracoscopic surgery ultrasound in vivo image of the nodule. 


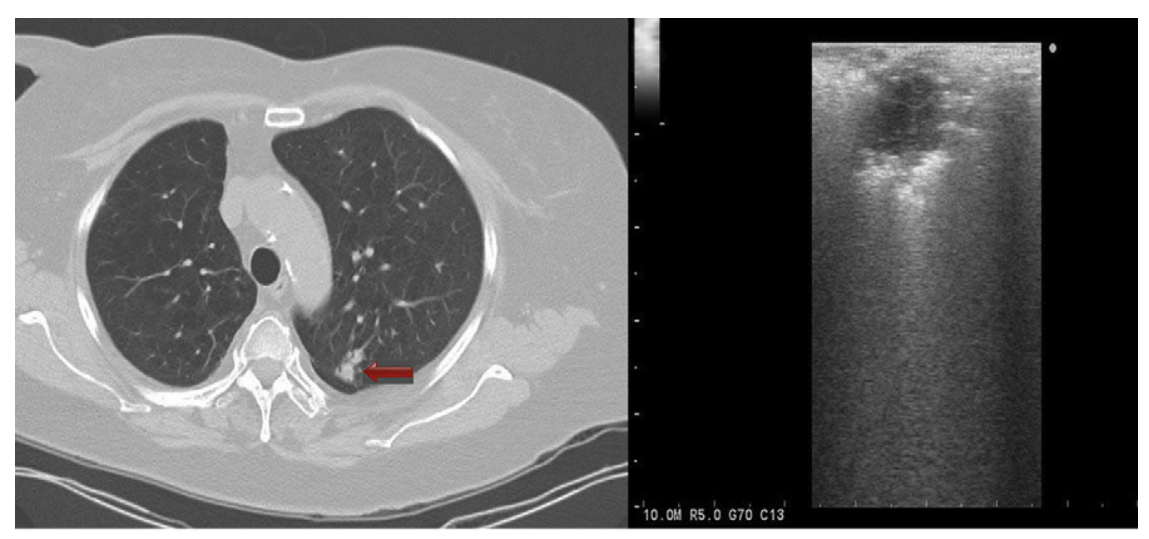

A

B

FIGURE 3. A, Preoperative computed tomography image of small, irregular, left upper lobe ground glass opacity with solitary components (arrow). B, Corresponding video-assisted thoracoscopic surgery ultrasound in vivo image of the nodule.

study, was not associated with any morbidity. Furthermore, VATS-US does not require the adaptation or elongation of standard VATS incisions in any way.

The present study was not designed to demonstrate the superiority of VATS-US compared with invasive preoperative localization techniques (eg, hook wire insertion, methylene blue, bronchoscopic nodule tagging). It was designed to demonstrate the sensitivity of VATS-US compared with standard VATS techniques without preoperative localization and to evaluate the ability of VATS-US to prevent conversion to thoracotomy in patients with nodules not localizable using standard VATS techniques.

Although preoperative methods of nodule localization have been shown to be effective and relatively safe in several studies, they are still not devoid of risk. Reported complications have included pneumothorax and pulmonary hemorrhage. ${ }^{6,13,14}$ Technical difficulties can lead to failure of hook wire placement. The wire can also be misplaced (improper insertion placement). ${ }^{15}$ The site of methylene blue injection can be far from the site of the nodule, or, more commonly, the stain is either not visible after injection or has spread and stained a large portion of normal lung surrounding the lesion, preventing nodule localization. ${ }^{10}$ Migration of the coil in the lung parenchyma during the period between coil insertion and operative resection can occur in $3 \%$ to $10 \%$, leading to failure of localization if the displacement is more than $10 \mathrm{~mm}$ from the nodule of concern. ${ }^{9,16,17}$

In completely deflated lungs, the structures of the pulmonary arterioles and venules are identified as homogeneous hypoechoic areas, and the bronchioles appear as hyperechoic spots, in the vicinity of the pulmonary arterioles. The nodule of interest is identified by US visualization of a hyper- or hypoechoic nodule or by visualization of a hyperechoic shadow beneath the nodule. This technique can be performed without the use of any other identifying methods and is less cumbersome and expedient. ${ }^{18}$
Using the high-quality imaging of US guidance to identify small and deep nodules, a strong correlation exists between resection margins measured using US, with the margins verified by histologic examination in the resected lung specimens. ${ }^{18}$

Intracavitary US has been shown to be able to identify very small pulmonary nodules down to $4 \mathrm{~mm}$ in diameter and even nodules deeply situated in the lung parenchyma. ${ }^{19}$ In the present study, we were able to localize small nodules, down to $2 \mathrm{~mm}$ using US measurement. The detection rate of pulmonary nodules has been shown to be much greater using VATS-US compared with video visualization or finger palpation. ${ }^{20,21}$ This was confirmed in the present study, with $27 \%$ of lung nodules detected by VATS visualization and $40 \%$ by finger palpation. This compares with $93.5 \%$ of lung nodules detected using VATS-US examination.

The efficacy of US is manifested by comparison with the number and size of the nodules identified using highresolution CT images preoperatively. Furthermore, in 1 study, US could identify 2 new small nodules not identified before using spiral $\mathrm{CT}^{22}$ In the present study, we were unable to localize any nodules not identified on the preoperative CT scan. However, the goal was to find the nodule of interest and not other nodules; therefore, minimal time was spent searching for other nodules not identified on the preoperative CT scan.

Intracavitary US characteristics have been shown to correlate with probability of malignancy in published data. ${ }^{20-24}$ We were, however, unable to confirm these findings in the present study. This was probably secondary to the sample size in our study being too small to appreciate an association between the probability of malignancy and the US characteristics of the nodules.

VATS-US has been shown to be an extremely safe and effective method of pulmonary nodule detection. No associated complications have been reported with VATS-US. ${ }^{21}$ Our study has confirmed these findings. 
TABLE 1. Lesion size and corresponding distance from pleura

\begin{tabular}{|c|c|c|c|c|c|}
\hline \multirow[b]{2}{*}{ Finding } & \multicolumn{2}{|c|}{$\begin{array}{c}\text { VATS-US } \\
\text { measurement }(\mathbf{m m}) \\
\end{array}$} & \multirow{2}{*}{$\begin{array}{c}\text { CT scan } \\
\text { (distance from } \\
\text { pleura; } \mathbf{m m} \text { ) }\end{array}$} & \multicolumn{2}{|c|}{$\begin{array}{c}\text { Pathologic } \\
\text { measurement }(\mathbf{m m}) \\
\end{array}$} \\
\hline & Short axis & Long axis & & Short axis & Long axis \\
\hline Positive & 2 & 3.7 & 14 & 6 & 6 \\
\hline Positive & 2.3 & 2.7 & 3 & 4 & 8 \\
\hline Positive & 2.5 & 5.6 & 1.3 & 8 & 8 \\
\hline Positive & 2.9 & 3.2 & 2 & 8 & 10 \\
\hline Positive & 2.9 & 15.8 & 1 & 8 & 15 \\
\hline Positive & 3.1 & 5.8 & 3 & 4.5 & 4.5 \\
\hline Positive & 3.6 & 5 & 7.5 & 5 & 7 \\
\hline Positive & 3.6 & 4.2 & 1 & 5 & 5 \\
\hline Positive & 3.6 & 5.1 & 18 & 24 & 25 \\
\hline Positive & 4.7 & 5.7 & 9.6 & 8 & 8 \\
\hline Positive & 5.4 & 8.8 & 15.3 & 8 & 8 \\
\hline Positive & 6.3 & 6.4 & 20 & 6 & 6 \\
\hline Positive & 6.9 & 9.4 & 24.1 & 16 & 18 \\
\hline Positive & 7.6 & 7.8 & 1.4 & 10 & 10 \\
\hline Positive & 7.6 & 9.4 & 4 & 10 & 13 \\
\hline Positive & 7.8 & 8 & 2 & 5 & 6 \\
\hline Positive & 8 & 8 & 14.5 & 6 & 7 \\
\hline Positive & 8.3 & 15.7 & 1 & 12 & 12 \\
\hline Positive & 8.5 & 9.4 & 23.1 & 10 & 15 \\
\hline Positive & 8.5 & 11 & 10.6 & 20 & 22 \\
\hline Positive & 8.6 & 9.1 & 4.7 & 8 & 8 \\
\hline Positive & 8.6 & 8.7 & 5 & 10 & 10 \\
\hline Positive & 8.7 & 14 & 1 & 13 & 13 \\
\hline Positive & 8.8 & 8.9 & 1 & 15 & 20 \\
\hline Positive & 8.9 & 15.8 & 5.4 & 14 & 16 \\
\hline Positive & 8.9 & 10.2 & 8.7 & 10 & 10 \\
\hline Positive & 9 & 6.4 & 2 & 10 & 10 \\
\hline Positive & 9.1 & 15.4 & 1 & 11 & 11 \\
\hline Positive & 9.4 & 15.7 & 6.5 & 15 & 16 \\
\hline Positive & 10.1 & 12.6 & 5 & 8 & 15 \\
\hline Positive & 10.8 & 12.24 & 14 & 15 & 15 \\
\hline Positive & 10.8 & 13 & 13 & 15 & 20 \\
\hline Positive & 11.1 & 11.9 & 4.6 & 15 & 18 \\
\hline Positive & 11.5 & 13.4 & 15 & 13 & 17 \\
\hline Positive & 12.1 & 14.3 & 10.9 & 15 & 21 \\
\hline Positive & 12.1 & 14.1 & 14.7 & 10 & 11 \\
\hline Positive & 12.3 & 15.9 & 15.7 & 15 & 18 \\
\hline Positive & 12.3 & 24.8 & 12.5 & 25 & 30 \\
\hline Positive & 12.9 & 16.3 & 4.2 & 11 & 13 \\
\hline Positive & 14.1 & 14.7 & 12.2 & 15 & 20 \\
\hline Positive & 14.4 & 15.5 & 11 & 10 & 18 \\
\hline Positive & 19.2 & 25.5 & 9.5 & 20 & 62 \\
\hline Positive & 20.4 & 27.7 & 3 & 10 & 15 \\
\hline Negative & NA & NA & 17.6 & 8 & 7 \\
\hline Negative & NA & NA & 10.6 & 1 & 5 \\
\hline Negative & NA & NA & 9 & 2 & 2 \\
\hline
\end{tabular}

VATS-US, Video-assisted thoracoscopic surgery ultrasonography; $C T$, computed tomography; $N A$, not available.

Intracavitary US by a radiologist during VATS has been shown by others to be short (approximately 7.5 minutes). However the logistical issues of waiting for a radiologist and the time it takes for the radiologist to come to the
TABLE 2. VATS-US results versus final pathologic results for nodules of interest

\begin{tabular}{lcc}
\hline & \multicolumn{2}{c}{ Pathologic finding } \\
\cline { 2 - 3 } VATS-US result & Positive & Negative \\
\hline Positive & $43(\mathrm{TP})$ & $0(\mathrm{FP})$ \\
Negative & $3(\mathrm{FN})$ & $0(\mathrm{TN})$ \\
\hline
\end{tabular}

VATS-US, Video-assisted thoracoscopic surgery ultrasonography; $T P$, true positive; $F P$, false positive; $F N$, false negative; $T N$, true negative.

operating room make this option less desirable. ${ }^{25}$ This can be avoided if the surgeon is the ultrasonographer. US experience is not a necessary prerequisite for performing VATSUS, and 4 different thoracic surgeons and multiple residents and fellows localized the nodules in the present study. VATS-US is a simple technique, with a rapid learning curve, manifested by a rapid decrease in the time necessary for the US examination after the first few cases to reach an average of 4 minutes.

The most important finding in the use VATS-US in the present study was its role in preventing conversions from VATS to open thoracotomy. In 20 cases, lung nodules were not identifiable using any of the traditional techniques and were identified only with VATS-US. VATSUS, therefore, prevented conversion to thoracotomy or lobectomy without tissue diagnosis in $43.5 \%$ of the cases.

\section{CONCLUSIONS}

Surgeon-performed intracavitary VATS-US offers a real-time, simple, and effective technique for localization of difficult to visualize nonsubpleural pulmonary nodules. This technique is completely safe and without any complications. Moreover, it can prevent imminent conversion to open thoracotomy during selected VATS wedge resection.

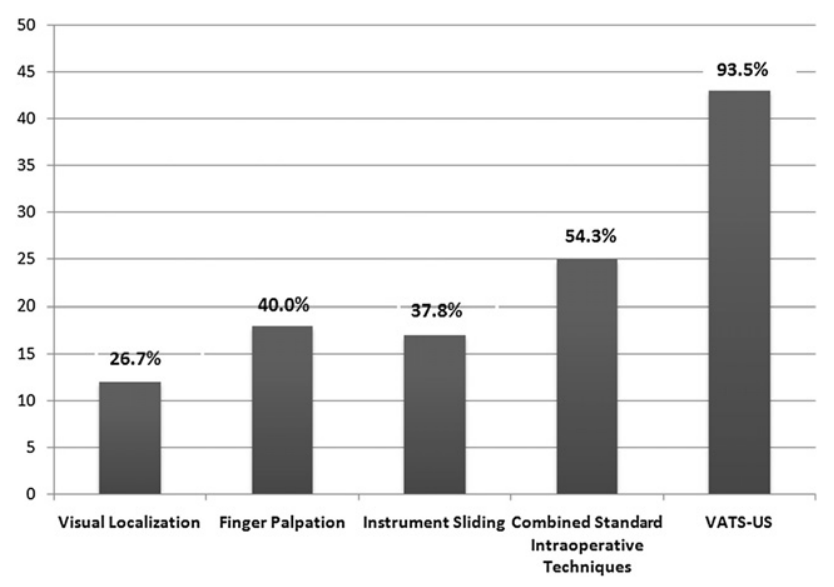

FIGURE 4. Comparison of video-assisted thoracoscopic surgery localization technique's ability to identify nodules of interest. 
TABLE 3. Ultrasound characteristics of nodules examined using VATS-US and their relationship to pathologic nature of nodule

\begin{tabular}{lcc}
\hline Characteristic & Malignant & Benign \\
\hline Shape & 10 & \\
$\quad$ Round & 11 & 8 \\
Oval & 8 & 2 \\
$\quad$ Irregular & & 4 \\
Borders & 16 & 6 \\
$\quad$ Ill defined & 13 & 8 \\
$\quad$ Well defined & & \\
Echogenicity & 3 & 1 \\
$\quad$ Hyperechoic & 9 & 5 \\
Medium & 17 & 8 \\
Hypoechoic & & 1 \\
Doppler & 5 & \\
Positive &
\end{tabular}

VATS-US, Video-assisted thoracoscopic surgery ultrasonography.

\section{References}

1. Kuo E, Bharat A, Bontumasi N, Sanchez C, Zoole JB, Patterson GA, et al. Impact of video-assisted thoracoscopic surgery on benign resections for solitary pulmonary nodules. Ann Thorac Surg. 2012;93:266-72.

2. Dowling RD, Landreneau RJ, Miller DL. Video-assisted thoracoscopic surgery for resection of lung metastasis. Chest. 1998;113:2-5S.

3. Powell TI, Jangra D, Clifton JC, Lara-Guerra H, Church N, English J, et al. Peripheral lung nodules: fluoroscopically guided video-assisted thoracoscopic resection after computed tomography-guided localization using platinum microcoils. Ann Surg. 2004;240:481-8.

4. Suzuki K, Nagai K, Yoshida J, Ohmatsu H, Takahashi K, Nishimura M, Nishiwaki Y. Video-assisted thoracoscopic surgery for small indeterminate pulmonary nodules: indications for preoperative marking. Chest. 1999;115:563-8.

5. Cardillo G, Regal M, Sera F, et al. Videothoracoscopic management of the solitary pulmonary nodule: a single-institution study on 429 cases. Ann Thorac Surg. 2003;75:1607-12.

6. Ciriaco A, Negri A, Puglisi A, Nicoletti B, Del Maschio B, Zannini A. Video-assisted thoracoscopic surgery for pulmonary nodules: rationale for preoperative computed tomography-guided hookwire localization. Eur J Cardiothorac Surg. 2004;25:429-33.

7. Partrick DA, Bensard DD, Teitelbaum DH, Geiger JD, Strouse P, Harned RK. Successful thoracoscopic lung biopsy in children utilizing preoperative CTguided localization. J Pediatr Surg. 2002;37:970-3.

8. Nomori H, Horio H, Naruke T, Suemasu K. Fluoroscopy-assisted thoracoscopic resection of lung nodules marked with lipiodol. Ann Thorac Surg. 2002;74: 170-3.

9. Miyoshi T, Kondo K, Takizawa H, Kenzaki K, Fujino H, Sakiyama S, et al. Fluoroscopy-assisted thoracoscopic resection of pulmonary nodules after computed tomography-guided bronchoscopic metallic coil marking. J Thorac Cardiovasc Surg. 2006;131:704-10.

10. Wang YZ, Boudreaux JP, Dowling A, Woltering EA. Percutaneous localisation of pulmonary nodules prior to video-assisted thoracoscopic surgery using methylene blue and Tc-99. Eur J Cardiothorac Surg. 2010;37:237-8.

11. Daniel TM, Altes TA, Rehm PK, Williams MB, Jones DR, Stolin AV, et al. A novel technique for localization and excisional biopsy of small or ill-defined pulmonary lesions. Ann Thorac Surg. 2004;77:1756-62.

12. Chella A, Lucchi M, Ambrogi MC, Menconi G, Melfi FM, Gonfiotti A, et al. A pilot study of the role of Tc-99 radionuclide in localization of pulmonary nodular lesions for thoracoscopic resection. Eur J Cardiothorac Surg. 2000;18:17-21.

13. Saito H, Minamiya Y, Matsuzaki I, Tozawa K, Taguchi K, Nakagawa T, et al. Indication for preoperative localization of small peripheral pulmonary nodules in thoracoscopic surgery. J Thorac Cardiovasc Surg. 2002;124:1198-202.

14. Wicky S, Mayor B, Schnyder P. Methylene blue localizations of pulmonary nodules under CT-guidance: a new procedure used before thoracoscopic resections. Int Surg. 1997;82:15-7.

15. Morita R, Kaneko K, Suga M, Omoto R. Computed tomography-guided hookwire localization of small pulmonary nodules for thoracoscopic biopsy. Nihon Kokyuki Gakkai Zasshi. 1999;37:439-42.
16. Mayo JR, Clifton JC, Powell TI, English JC, Evans KG, Yee J, et al. Lung nodules: CT-guided placement of microcoils to direct video-assisted thoracoscopic surgical resection. Radiology. 2009;250:576-85.

17. Krüger K, Eyl G, Morgenroth C, Schneider P, Hoelscher A, Lackner K. Success and complication rate of CT-guided marking of pulmonary nodules with coil wires for video-assisted thoracoscopic surgery (VATS). Rofo. 2006;178:1250-4

18. Kondo R, Yoshida K, Hamanaka K, Hashizume M, Ushiyama T, Hyogotani A, et al. Intraoperative ultrasonographic localization of pulmonary ground glass opacities. J Thorac Cardiovasc Surg. 2009;138:837-42.

19. Gruppioni F, Piolanti M, Coppola F, Papa S, Di Simone M, Albini L, et al Intraoperative echography in the localization of pulmonary nodules during video-assisted thoracic surgery. Radiol Med. 2000;100:223-8.

20. Matsumoto S, Hirata T, Ogawa E, Fukuse T, Ueda H, Takashi K, et al. Ultrasonographic evaluation of small nodules in the peripheral lung during videoassisted thoracic surgery (VATS). Eur J Cardiothorac Surg. 2004;26:469-73.

21. Sortini D, Feo V, Carcoforo P, Carrella G, Pozza E, Liboni A, et al. Thoracoscopic localization techniques for patients with solitary pulmonary nodule and history of malignancy. Ann Thorac Surg. 2005;79:258-62.

22. Piolanti M, Coppola F, Papa S, Pilotti V, Mattioli S, Gavelli G. Ultrasonographic localization of occult pulmonary nodules during video-assisted thoracic surgery. Eur Radiol. 2003;13:2358-64.

23. Hamazaki N. Clinical application of power Doppler sonography for the qualitative diagnosis of subpleural lesions. J Nara Med Assoc. 1999;50:497-508.

24. Mathis G. Thorax sonography part II: peripheral pulmonary consolidation. Ultrasound Med Biol. 1997;23:1141-53.

25. Greenfield A, Steiner R, Liu B, Cohn H, Goldberg BB, Rawool M, et al. Sonographic guidance for the localization of peripheral pulmonary nodules during thoracoscopy. Am J Radiol. 1997;168:1057-60.

\section{Discussion}

Dr Bryan Fitch Meyers (St Louis, Mo). I enjoyed your presentation and I have a couple of questions to stimulate discussion.

You mentioned that US was used in each case here, but in the range of sizes of the lesions you described, I saw a 51-mm lesion and a $27-\mathrm{mm}$ lesion. I would argue that you really do not need US to find a $5-\mathrm{cm}$ or a nearly $3-\mathrm{cm}$ lesion in the lung. In this case, you were trying to use it broadly and might have used it in many instances in which it absolutely was not needed. Can you comment on that?

Dr Khereba. There was only 1 case with a 51-mm nodule; actually, it was found to be a bronchogenic cyst. According to the surgeon and because of the centrality of this nodule, he thought that it might be difficult to find this nodule using finger palpation or instrument sliding. It was not visible during the study, but it was found by finger palpation and instrument sliding, and US as well. For the other nodules, the $20 \mathrm{~cm}$ or $27 \mathrm{~cm}$, that was 2 or 3 cases in the study, and they were deeper into the lung parenchyma.

We chose our nodules according to the choice of the surgeon. We revised the nodules; however, if the surgeon needs US to be performed, he asks for the US.

Dr Charles R. Bridges (Charlotte, $N C$ ). It is important to point out that the mean diameter long axis was only $13 \mathrm{~mm}$. So, that 51 $\mathrm{mm}$ was clearly an outlier.

Dr Khereba. It is only 1 nodule that has been passing our mean; most of the nodules were between 5 and $50 \mathrm{~mm}$.

Dr Meyers. I agree, and I understand that you are making a point here and you are demonstrating the potential utility of a new technology, but in terms of actual clinical use, if the mean is $13 \mathrm{~mm}$, that means one half of the lesions are larger than that, and generally the challenge is with the subcentimeter lesion. Therefore you would argue that perhaps one half of these lesions used in this demonstration trial would really be a challenge to find. So, the broad utility of something like this might need to be focused slightly more on the most challenging lesions. 
Dr Khereba. Thank you for your question. I think that in published studies, it was proven that the conversion rate is greater for lesions either less than $10 \mathrm{~mm}$ or more than $1 \mathrm{~cm}$ in the depth of the lung.

Thank you.

Dr Chumy Nwogu (Buffalo, NY). I have 3 questions for you. This was a very interesting presentation, and I am looking forward to using it in my practice. However, I was wondering what the source of the data on the other techniques is, whether you were just comparing your US findings with results from previously published studies. Were you studying the comparative results of these techniques in your own patients with similar lesion sizes, a similar depth from the surface?

Dr Khereba. Yes, exactly. You mean the intraoperative finger palpation and instrument sliding?

Dr Nwogu. Yes.

Dr Khereba. For every case, we performed finger palpation, instrument sliding, and VATS-US.

Dr Nwogu. I was wondering whether you had some patients with very severe chronic obstructive pulmonary disease in whom the lung was not completely deflated. In those cases, was it more difficult for you to apply the US in that situation?

Dr Khereba. We did not have any difficulty with emphysematous patients. We had 1 case actually in which we could not achieve complete lung deflation, and we still found the nodule using US.
Dr Nwogu. So the air does not interfere at all with your visualization?

Dr Khereba. It is more difficult to find the nodules in emphysematous patients. However, all our population that was examined, most of them were already emphysematous, but we did not have difficulty with that.

Dr Nwogu. There were about 3 cases in which you could not localize the lesion using your technique. What do you think were the causes of failure in those cases?

Dr Khereba. We had 3 cases. The first case was a patient in whom there were multiple pulmonary nodules and we could not find the nodules using any other method, and it was impossible to examine the lung because it was not echogenic at all. Thus, because of the multiple pulmonary nodules, we performed a blind incision and VATS resection, and we found a nodule. Finally, it turned out to be Hodgkin's lymphoma.

The other nodule was difficult to find, because it was so posterior into the lung and proximal to the spine, and we could not find it using US in this position. Finally, because of its high probability on positron emission tomography scan and CT scan, we performed a lobectomy on this patient, and we found the nodule in vitro using US. However, we still considered that as a negative result.

The third nodule was examined using US, and we could not find the nodule, but we still found it by finger palpation. We readministered US on the nodule, and we could not find it. So, we finally considered that a negative result as well. 